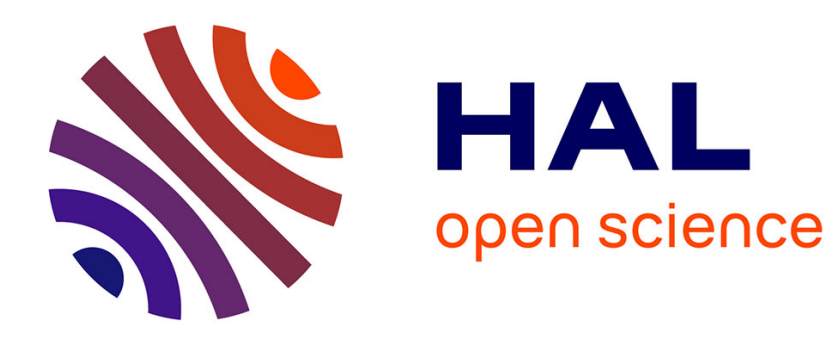

\title{
La prédication seconde en français: essai de mise au point
}

\author{
Eva Havu, Michel Pierrard
}

\section{To cite this version:}

Eva Havu, Michel Pierrard. La prédication seconde en français: essai de mise au point. Travaux de Linguistique: Revue Internationale de Linguistique Française, 2008, pp.7-21. halshs-00454957

\section{HAL Id: halshs-00454957 https://shs.hal.science/halshs-00454957}

Submitted on 9 Feb 2010

HAL is a multi-disciplinary open access archive for the deposit and dissemination of scientific research documents, whether they are published or not. The documents may come from teaching and research institutions in France or abroad, or from public or private research centers.
L'archive ouverte pluridisciplinaire HAL, est destinée au dépôt et à la diffusion de documents scientifiques de niveau recherche, publiés ou non, émanant des établissements d'enseignement et de recherche français ou étrangers, des laboratoires publics ou privés. 


\section{La prédication seconde en français : essai de mise au point}

Eva Havu \& Michel Pierrard

«Cruciale pour la philosophie du langage à l'anglo-saxonne, bien présente sous le nexus des Danois et revenant en force dans les small clauses 'clausules' de la linguistique américaine, la prédication (...) a été incroyablement sous-estimée par la grammaire française » constate Marc Wilmet dans sa Grammaire critique du français (2007, §644 : 572). Le constat était particulièrement pertinent pour la prédication seconde, du moins jusqu'à la sortie, il y a 20 ans, sous la direction de Ludo Melis, d'un numéro thématique de Travaux de Linguistique (17/1988) consacré à cette notion. A juste titre, ce numéro constitue aujourd'hui encore un jalon pour les études sur la question, dans la mesure où, pour la première fois, il proposait la prédication seconde comme un cadre d'analyse permettant d'appréhender une large série de constructions. Dès son introduction, il esquissait les contours d'une définition de la catégorie et les études proposées sur une large série de tours relevaient certaines constantes, syntaxiques, sémantiques ou pragmatiques, qui pouvaient leur être attribuées, et qui justifiaient leur regroupement sous l'étiquette de prédication seconde.

Depuis lors, la prédication seconde a fait l'objet de diverses tentatives de définition et de nombreuses études sur des tours particuliers qui peuvent y être rattachés. Nous songeons particulièrement ici au numéro thématique de Langue française (127/2000), sous la direction de Pierre Cadiot et Naoyo Furukawa, mais bien d'autres linguistes se sont attaqués à la question (p.ex. Forsgren, 2000, Neveu, 2000, 1998, Combettes, 1998, Wilmet, 1997, 2007, Furukawa, 1996...). Le moment nous semblait donc propice pour refaire une mise au point sur l'exploitation en linguistique française de la prédication seconde.

1. L'examen des principales contributions sur la question nous permet de dégager une série de traits récurrents de la prédication seconde (dorénavant PRED2). Ils peuvent être regroupés selon les trois propriétés suivantes, qui synthétisent chaque fois plusieurs traits : 
i) l'instauration d'un lien prédicatif de type attributif entre un thème et un prédicat (trait $a$ ) mais sans marque verbale de prédication (trait $b$ ) : «formant un prédicat second, de type attributif, mais non fini au plan syntacticosémantique » (Neveu 2000), « exprime sémantiquement un contenu phrastique » (Cadiot \& Furukawa, 2000), «Greffée sur un terme quelconque de la prédication première » (Wilmet, 2007, §639: 562) ;

ii) un rapport de dépendance de PRED2 envers une prédication supérieure (trait a), la prédication seconde étant syntaxiquement intégrée dans la prédication régissante (trait $b$ ) : « une relation prédicative intégrée dans une prédication à un niveau supérieur » (Melis, 1988) ; «statut syntaxiquement intégré » (Cadiot \& Furukawa, 2000) ;

iii) position périphérique de PRED2 par rapport à la structure argumentale de l'énoncé (trait a) et apport de sens facultatif (trait b) : «occupant une position périphérique à la structure argumentale de l'énoncé » (Neveu, 2000 : 121) ; «qui ne constitue pas le foyer de la visée phrastique » (Mélis, 1988); «facultative [...], surajoutée à l'énoncé » (Wilmet, 1997 : 420).

La première propriété concerne la syntaxe interne de la prédication seconde, qui implique l'instauration d'un lien prédicatif (trait 1a) et l'absence d'une forme verbale (conjuguée) (trait 1b). La seconde propriété définitoire est par contre orientée vers la syntaxe externe de PRED2, et se traduit par l'instauration d'un rapport de dépendance de préd2 envers la prédication régissante (trait 2a) et l'intégration syntaxique de PRED2 dans celle-ci (trait $2 b$ ). Enfin, la troisième propriété définitoire évoque le caractère accessoire de PRED2. Elle se concrétise par la position périphérique de PRED2 par rapport à la structure argumentale de l'énoncé (trait 3a) et par un apport de sens facultatif de PRED2 (trait 3b).

D'autre part, le terme de prédication seconde est utilisé pour désigner une très large gamme de constructions apparemment assez disparates. La liste des tours présentés ici comme des PRED2 reprend l'ensemble des cas reconnus depuis 1988 par les linguistes français travaillant 
sur la construction (cf. en particulier Cadiot \& Furukawa, 2000 et Melis, 1988). Ils sont répertoriés selon un critère déjà utilisé par Melis en 1988 :

\section{Type 1. prédications associées à l'énoncé}

1.1. Appositions de phrase

[1]

Il est parti; décision malheureuse.

1.2. Compléments de phrase «évaluatifs »

Malheureusement, il est parti.

\subsection{Compléments absolus}

[3] a) La tête dans les nuages, il répondit pourtant avec aplomb.

b) Sa nièce arrivant, c'était le feu dans la maison.

\subsection{Infinitifs de narration}

[4]

Je m'écriai : «voilà notre homme! » et l'assistance d'applaudir, (...). (Chateaubriand;

Grevisse Gosse, 1991, § $871: 1314$ )

\section{Type 2. prédications associées à un groupe nominal}

\subsection{Appositions}

[5] a) J'ai visité Paris, ville des sciences et des arts.

b) Sartre, mon auteur préféré, se vend mal.

2.2. Adjoints détachés

[6] a) Jean est parti, furieux.

b) Regardant à sa gauche, il vit le facteur qui sortait de sa maison.

c) Reposé et de bonne humeur, il est allé travailler.

d) $\quad \mathrm{X}$ est mort mercredi, en sortant de sa maison pour aller...

e) Au bureau de poste principal, rue Saint-François-de-Paul, on inaugurait hier..

2.3. Associations indirectes ( $N$ de $N$ : cf. Gaatone, 1988 ; $N$ de Adj : cf. Cadiot et Furukawa, 2000)

[7] a) Ce fripon de valet m'a à nouveau volé.

b) Il y a encore une place de libre. 
2.4. Prédicats ayant la forme d'une proposition subordonnée : il s'agit a) de relatives explicatives [8], b) de complétives apposées [9] et c) d'infinitives apposées [10] :

[8] Les hommes, qui étaient regroupés dans la cour, furent massacrés dans la matinée.

[9] a) Je n'ai qu'un souhait : qu'il vienne demain.

b) L'idée qu'elle parte le désespérait.

[10] a) Il n'a qu'une idée : s'évader.

b) L'idée de devoir partir le désespérait.

\section{Type 3. prédications associées à certaines fonctions syntaxo-sémantiques}

\subsection{Attributs directs de l'objet}

[11] a) Je m'appelle Socrate.

b) J'aime le café chaud.

c) Paul trouve la mesure indispensable.

d) Luc rend Marie heureuse.

\subsection{Emplois attributifs d'avoir}

[12] a) Il a les yeux bleus.

b) Cette année, notre village a ses vendanges plutôt réussies.

\subsection{Attributs indirects de l'objet}

[13] a) On le traite de nigaud.

b) Il considère cette affaire comme importante.

3.4. Propositions a) relatives et $b$ ) infinitives après un objet d'un verbe de perception

[14] a) J'ai vu Paul qui fumait / J'entends un bébé qui pleure

b) J'ai vu Paul fumer/J'entends un bébé pleurer.

3.5. Pseudo-relatives (Furukawa, 1996)

[15] a) Elle est là qui pleure.

b) J'ai la tête qui tourne.

c) Et ma tante qui n'arrive pas!

3.6. Vocatifs

[16] Que désirez-vous, Madame? 
3.7. Constructions avec complément prépositionnel (Cadiot, 2000)

Paul m'a séduit par ses bonnes manières.

2. Le tableau récapitulatif ci-dessous indique, à partir des observations de Havu \& Pierrard 2004, en quelle mesure les quinze tours identifiés ci-dessus expriment les six traits révélant les propriétés basiques attribuées à la prédication seconde. Cette comparaison systématique permet de déterminer le degré d'adéquation des différentes constructions avec les propriétés basiques relevées :

\begin{tabular}{|c|c|c|c|c|c|c|}
\hline & trait 1a & trait $1 b$ & trait $2 a$ & trait $2 b$ & trait 3a & trait $3 b$ \\
\hline \multicolumn{7}{|l|}{ Type 1} \\
\hline tour 1.1 & - & + & - & - & + & + \\
\hline tour 1.2 & - & + & - & - & + & + \\
\hline tour 1.3 & + & - & + & - & + & + \\
\hline tour 1.4 & + & - & - & - & + & + \\
\hline \multicolumn{7}{|l|}{ Type 2} \\
\hline tour 2.1 & + & + & + & - & + & + \\
\hline tour 2.2 & + & $+/-$ & + & - & + & $+/-$ \\
\hline tour 2.3 & - & + & + & $-/+$ & $-/+$ & $-/+$ \\
\hline tour 2.4 & - & $+/-$ & + & $-/+$ & + & $+/-$ \\
\hline \multicolumn{7}{|l|}{ Type 3} \\
\hline tour 3.1 & + & + & + & + & $-/+$ & - \\
\hline tour 3.2 & + & + & + & + & - & - \\
\hline tour 3.3 & - & + & + & + & - & - \\
\hline tour 3.4 & - & - & + & + & - & + \\
\hline tour 3.5 & - & - & + & - & + & $+/-$ \\
\hline tour 3.6 & - & + & + & - & + & + \\
\hline tour 3.7 & - & - & + & + & - & + \\
\hline
\end{tabular}

Dans l'hypothèse où les propriétés identifiées sont effectivement des caractéristiques prototypiques de PRED2, plusieurs constats remarquables découlent de l'analyse du tableau synthétique et peuvent nourrir la réflexion sur la délimitation du domaine de la prédication seconde : 
2.1. L'observation la plus marquante est sans doute qu'aucun tour ne répond pleinement à l'ensemble des traits avancés pour caractériser la construction mais que tous présentent au moins la moitié des traits : une définition uniforme de la prédication seconde paraît donc hasardeuse; une approche graduée s'impose avec des tours plus prototypiques jouxtant d'autres qui se situeront plus à la périphérie du domaine. De ce point de vue, la répartition des tours en trois grands types, proposés par Melis (1988), devrait sans doute être affinée. Ainsi, dans le cas du type 3, particulièrement hétérogène, une séparation plus nette pourrait être introduite entre un 'noyau dur' (les attributs de l'objet et l'emploi attributif d'avoir) et une zone plus excentrée (les pseudo-relatives et les constructions avec complément prépositionnel).

2.2. Le trait caractérisant le plus grand nombre de tours et qui par conséquent semble le plus global pour identifier la prédication seconde est le trait 2a, c'est-à-dire l'instauration d'un rapport de dépendance entre PRED2 et une autre prédication. Une autre caractéristique, le trait 3a, la position périphérique de PRED2, qui est centrale dans les définitions de concepts tels l'apposition (Neveu, 2000 : 120-121), la construction détachée (Combettes, 1998 : 13 15) ou l'adjoint (les 'free adjuncts' de Kortmann, 1991 : 6-7), est largement représentée et identifie parfaitement les prédications associées à l'énoncé (types 1) ou à un groupe nominal (type 2). Cependant, elle ne correspond guère au fonctionnement du 'noyau dur' des prédications associées à certaines fonctions syntaxo-sémantiques (type 3).

2.3. Les traits couvrant le moins bien l'ensemble du domaine de la prédication seconde sont les traits 1a (marquage de l'instauration d'un rapport prédicatif) et 2b (l'intégration syntaxique de PRED2 dans PRED1). La première caractéristique fonde pourtant le concept de 'small clause' (cf. Aarts, 1992 : 9, Williams, 1994 : 77), tandis que la seconde est partagée par l'ensemble des tours qui sont à l'origine de la notion de prédication seconde (attributs directs et indirects de l'objet; emploi attributif d'avoir), mais ces traits semblent peu marquer le fonctionnement des autres tours intégrés au domaine. 
2.4. Du point de vue des différents tours étudiés, il faut relever que les types 2.1 et 2.2, traditionnellement considérés comme des appositions, des adjoints ou des constructions détachées, répondent le plus largement aux caractéristiques avancées. Ils se situent à la croisée des différentes propriétés et donc au coeur du domaine. Notons que le seul trait qu'ils ne manifestent pas est l'intégration syntaxique dans PRED1, ce qui correspond d'ailleurs à une intuition communément partagée à propos du fonctionnement des adjoints ou des constructions détachées (cf. déjà l'observation 2.2).

2.5. Parmi les autres tours, les prédications associées à la fonction attribut de l'objet (types 3.1 à 3.3) s'opposent radicalement aux prédications associées à l'énoncé (type 1), les deux groupes se positionnant en quelque sorte aux deux extrémités du domaine : alors que ces derniers sont presque exclusivement caractérisés par le caractère accessoire de PRED2 (propriété basique 3), ce qui les singularise quelque peu dans l'ensemble des tours examinés, les premiers sont avant tout identifiés par le lien prédicatif sans verbe conjugué (propriétés 1) et l'intégration syntaxique dans PRED1 (propriété 2), les traits singularisant la syntaxe interne et externe de PRED2.

Bref, les propositions de caractérisation de la prédication seconde révèlent nettement deux orientations pour appréhender le fonctionnement commun des tours au sein du domaine de PRED2 : à partir de la reconnaissance d'une relation de dépendance entre deux prédications, un premier axe met l'accent sur l'instauration d'un rapport prédicatif qui reste à la périphérie de la prédication régissante ; un second axe par contre souligne l'intégration des deux relations prédicatives qui tendent à mener en fin de compte à la construction d'un prédicat complexe.

3. Par ailleurs, la détermination des propriétés basiques de PRED2, telle que nous l'avons synthétisée ci-dessus, ne peut aboutir sans poser aussi la question des limites de la prédication seconde. Cette discussion doit être menée sur plusieurs plans. 


\subsection{Prédication seconde et subordination}

Faut-il appréhender la prédication seconde comme un stade dans le processus de réduction (réduction d'autonomie) d'une proposition (subordonnée) ? Ainsi, dans le cas de génération de 'small clauses' (cf. Aarts, 1992) :

[18] a) J'estime que les nouvelles sont positives

b) J'estime les nouvelles positives.

La prédication de [18a] que les nouvelles sont positives ne sera pas considérée comme une prédication seconde, alors que les nouvelles positives dans [18b] l'est. Ou au contraire, ce processus de réduction n'est-il qu'une conséquence secondaire d'un certain type de fonction, comme les exemples [8-9] ou (14-15] semblent l'indiquer? La relation subordonnée/ prédication seconde demande incontestablement à être approfondie, voire repensée.

\subsection{Préd2 : macro- ou microsyntaxe?}

Peut-on identifier des relations prédicatives secondes dans des arguments du verbe régissant ou doit-on au contraire les limiter aux termes associés (cf. Blanche-Benveniste, 1983 et Blanche-Benveniste et alii, 1990 ; cf. aussi Berrendonner et Reichler-Béguelin, 1989 : syntaxe de rection / syntaxe de présupposition, et Eriksson, 1993 et Jespersen, 1937 pour la notion de «nexus ») ? Il n'est pas toujours aisé de trancher en la matière.

On ne considère certainement pas les attributs du sujet comme des prédications secondes. Toutefois, les verbes «occasionnellement attributifs » (cf. Riegel et alii, 1999 : 235-236) ne se comportent pas de la même manière qu'un verbe copule, dans la mesure où l'attribut ne peut pas être repris par un pronom personnel, trait typique pour les associés (BlancheBenveniste et alii, $1990: 99)$ :

[19] a) Il est content $>$ Il l'est .

b) Il rentre content $>*$ Il le rentre.

Ce trait rapproche ces tours de certaines constructions où la mise de l'adjectif en prédication seconde l'exclut de la pronominalisation (cf. Wilmet, 2007, § 641: 567, qui parle ici d'apposition) : 
[20] a) Mon collègue fatigué ne peut pas venir. > Il (= collègue fatigué) ne peut pas venir.

b) Mon collègue, fatigué, ne peut pas venir > Fatigué, il (= collègue) ne peut pas venir/ *Le, mon collègue ne peut pas venir.

De même, certains compléments prépositionnels font partie d'une PRED2 s'ils sont prévus dans la structure argumentale du verbe (cf. Cadiot, 2000: 112: Paul m'a séduit par ses bonnes manières; cf. aussi Aarts, 1992 : 21 : I want the dog out of the house). Mais où est alors la limite entre un complément prépositionnel faisant partie de la structure argumentale de PRED1 [17b] et un complément prépositionnel instaurant une PRED2 [17a] :

[17] a) Paul m'a séduit par ses bonnes manières. (Cadiot, $2000: 112$ )

b) Elle donne des fleurs à sa mère ?

La notion d'association n'est-elle en outre pas relative et ne faut-il pas distinguer dès lors divers niveaux d'association? Ainsi, dans un grand nombre de PRED2, l'association est marquée par l'intégration du thème de PRED2 dans la prédication régissante [21a]. Est-ce un critère décisif et doit-on dès lors écarter les compléments absolus [21b] des PRED2 ?

[21] a) Effondrée, elle attendait la sentence des juges.

b) Le dos courbé, elle attendait la sentence des juges.

Il n'y a pas d'unanimité parmi les linguistes sur la question (Combettes, 1998 : 22-24, Blanche-Benveniste, 1998, Borillo, à paraître).

D’ailleurs, certaines prédications averbales semblent très proches des prédications secondes détachées : la ponctuation forte suffit-elle pour distinguer une prédication seconde [22b] d'une prédication autonome [22a]? Ou peut-on vraiment les séparer par leurs propriétés informationnelles, comme l'évoquent Delorme \& Lefeuvre (2004) ?

[22] a) La dernière fois, une délégation de salariés est venue la voir. Inquiets.

b) La dernière fois, une délégation de salariés est venue la voir, inquiets. (Delorme \& Lefeuvre, $2004: 287$ )

\subsection{Préd2 et circonstants}

Certains types de circonstants présentent des fonctionnements proches de ceux des prédications secondes : faut-il radicalement distinguer les deux classes ? Dans ce contexte, l'appréhension du gérondif et du participe présent est une question délicate à traiter : D’après Combettes (1998 : 19), le fonctionnement des gérondifs permet de les classer parmi 
les constructions à prédication seconde. Neveu (2000:31) par contre s'y oppose, dans la mesure où il n'y perçoit pas d'incidence nominale.

Certaines constructions prépositionnelles, parfois associées à des adjectifs / des participes passés, peuvent aussi apparaître en fonction d'épithète ou d'attribut (Combettes, 1998 : 17 18) :

[23] a) Sans défaut/, Paul est un collaborateur apprécié / Paul est sans défaut / Un homme sans défaut.

b) Dans son affolement, Paul a oublié de fermer le gaz.

[23a-b] peuvent aussi être remplacées par un adjectif (Impeccable, X est../ Affolé, Paul [...]). Toutefois, Leeman (2000 : 20) soulève certains problèmes : dans le cas de [23b], le SP ne peut pas apparaître en fonction d'attribut : *Paul est dans son affolement. Relevons enfin la question des adjoints détachés locatifs dont certains peuvent, d'après Forsgren (2000 : 39 ff ; v. aussi Combettes, 1998 : 87-88), être comptés parmi les prédications secondes. Est-il possible de répertorier ces différents tours dans des catégories différentes ?

\subsection{Préd2 et Topicalisation / dislocation/ reformulation}

En quelle mesure faut-il distinguer la prédication seconde d'une part et divers types de restructurations focalisantes, topicalisantes ou simplement reformulantes de l'autre?

Les topicalisations ou dislocations, qui sont toujours détachées, peuvent être confondues avec des prédications secondes. Neveu (1998 : 69-70) et Combettes (1998: 14), donnent des arguments pour distinguer les premières des secondes. Dans les dislocations / topicalisations [24a], l'élément détaché est référentiel et il s'établit une coréférence entre l'expression référentielle et l'élément de reprise :

[24] a) Jean voit ton frère tous les jours > Ton frère, Jean le voit tous les jours.

b) Fier de posséder un vivier talentueux, le FC Nantais-Atlantique va toutefois devoir témoigner d'ambitions beaucoup plus grandes. (Le Figaro 15/05/00 : 30)

Si la prédication seconde de type appositif coordonne des énoncés en leur conservant une relative autonomie sémantique [24b], la dislocation les unifie en établissant une relation de dépendance bilatérale entre les constituants. 
Parfois, il est difficile de distinguer une apposition d'une coordination ou d'une reformulation :

[25] a) Philippe par contre je peux te proposer quelque chose qui te fera faire un entraînement complet- un travail de résistance [...]. (Blanche-Benveniste \& Caddéo 2000 : 61)

b) Au milieu de tout, [sic] cela, une figure très pâle, une jeune fille, habillée en noir. (Noailly $2000: 55$ )

Dans [25a], Blanche-Benveniste \& Caddéo (2000 : 60-69) parlent d'une «apposition à désignation multiple », qui est difficile à distinguer de la coordination asyndétique. Dans [25b] par contre, Noailly (2000 : 54-55) ne voit pas une apposition, mais elle considère que chaque GN concourt pour sa part à la désignation et qu'il faudrait parler d'une reformulation (paraphrasable par en d'autres termes, ou plutôt).

Bref, la réflexion concernant la prédication seconde devra traiter des problèmes 'externes' des limites de l'ensemble des tours regroupés sous l'étiquette de PRED2

- par rapport aux fonctions argumentales (prépositionnelles) de la prédication régissante d'une part,

- par rapport aux propositions subordonnées de l'autre,

Il s'agit en outre là de questions qui jouent aussi un rôle crucial dans la définition 'interne' de PRED2, dans la caractérisation des propriétés censées délimiter des traits communs au domaine. Cela renvoie plus spécifiquement à la tension entre

- une appréhension en termes de fonction périphérique de la prédication de base (fonction 'apposée, voire 'détachée')

- et une autre qui voit plutôt une construction d'une prédication complexe à travers l'imbrication de deux rapports prédicatifs (prédications première et seconde).

\subsection{Problématique traitée}

Des sept articles, deux (Goes et Halmøy) comparent plusieurs types au sein du domaine de la prédication seconde, tout en les rapportant à d'autres constructions voisines. Les cinq autres contributions (Caddéo, Corminboeuf, Lagae, Larrivée, Tobback) choisissent plutôt d'affiner la description et l'interprétation d'un tour particulier. 
Jan Goes étudie le continuum sémantique qui s'établit entre l'attribut de l'objet facultatif, l'attribut du sujet facultatif, l'apposition et le complément circonstanciel. Les caractéristiques communes des adjectivaux attribut de l'objet facultatif et attribut du sujet facultatif l'ont amené à considérer ce dernier comme faisant partie intégrante de la prédication seconde. Un même continuum sémantico-pragmatique mène de ces deux types d'attribut à l'apposition adjectivale, qui se rapproche pour sa part du complément circonstanciel (adjectif invarié). L’introduction de deux critères nécessaires, mais non suffisants pourrait, d'après lui, être une solution permettant de séparer la prédication seconde du complément circonstanciel : l'incidence nominale et la prédication de type attributif (cf. propriété 1).

Odile Halmøy compare le gérondif et le participe présent, formes verbales non autonomes, qui sont subordonnées syntaxiquement à une prédication première ou à un élément de cette prédication et qui doivent pour cette raison et pour leur nature même de prédicat être considérés comme des représentants de la prédication seconde. Toutefois, les gérondifs et les participes ne forment pas des ensembles homogènes et peu différenciés. L'auteur se propose ici de cerner davantage, en l'illustrant par des exemples, le comportement spécifique des [FV-ant], selon leur fonction syntaxique, leur incidence nominale, verbale, endophrastique ou exophrastique -, leur place par rapport à leur support, leur caractère lié ou détaché, leur apport informationnel et leur rôle discursif. Si le participe présent peut former une construction à prédication seconde dans les types (1) (Compléments absolus), (2) (Adjoints détachés) et (3) (Attributs directs et indirects de l'objet), le gérondif apparaît dans une structure à prédication seconde seulement dans le type (2).

Dans sa contribution, Sandrine Caddéo réunit sous le terme «apposition »un certain nombre de constructions à comportement apparemment semblable de par leur rattachement à un «support » $(\mathrm{GN}$, pronom personnel disjoint, pronom indéfini) dans l'énoncé, mais qui n'y sont pas tous liées par la propriété de prédication seconde. En plus d'appositions adnominales («apposés nus »), elle traite de constructions dont la relation sémantique avec le support est pris en charge par une série d'éléments à statut varié, des apposés (ou 
«greffes ») à relais qui sont des organisateurs de relations (sauf, notamment, dont...). Ces constructions ne peuvent pas toutes être considérés comme des prédications secondes dans le sens que le lien entre le support et l'apposé s'établit par les effets sémantiques qu'ils apportent : focalisation, partition, effet paradigmatisant, etc. L'auteur approfondit donc la réflexion sur les limites du type 2.1. et sur ce qui peut être considéré comme une apposition.

Els Tobback a choisi d'étudier les constructions à attribut de l'objet, et se demande si toutes ces constructions peuvent être considérées comme des structures à prédication seconde : le verbe occupe-t-il le statut de verbe recteur fort, régissant la relation prédicative établie entre l'objet et l'attribut de l'objet de la proposition ou a-t-il tendance à perdre ce statut (verbe à rection faible), auquel cas les structures étudiées ne se laisseraient plus réellement qualifier de structures à prédication seconde? Elle arrive à la conclusion que l'analyse des verbes faibles paraît jusqu'à un certain point applicable dans certaines structures syntaxiques particulières, mais elle ne semble pas généralisable à l'ensemble de la structure attributive : les structures à $\mathrm{AO}$ semblent alterner entre un fonctionnement en tant que structures à prédication seconde et un fonctionnement où la prédication «seconde » tend à devenir la prédication principale de l'énoncé, ce qui devrait être mieux pris en considération dans la descriptions des constructions du type 3.

Dans quelle mesure les participes passés peuvent-ils être utilisés dans la construction existencielle de + forme adjectivante, se demande Véronique Lagae. Le choix des adjectifs et des participes passés est fortement contraint dans cette construction, et son étude met en évidence un certain nombre de propriétés qui opposent les deux séries de formes. Elle constate qu'en comparaison avec les adjectifs, les participes passés, qui sont d'origine verbale, sont soumis à des contraintes de nature différente, essentiellement aspectuelle : seuls sont acceptables les participes passés de verbes processifs téliques pouvant constituer une forme résultative avec être ( $L$ 'article est terminé). A cela s'ajoutent d'autres restrictions plus spécifiques. Ces observations montrent que le type 2.3. doit être considéré comme un type avec de multiples contraintes.

Gilles Corminboeuf traite d'un type de construction relative qui n'a pas été pris en compte dans le relevé que nous avons établi des constructions à prédication seconde : les 
tours en $\{d e+$ adjectif $\}$ qui comprennent souvent une proposition relative (type De tendres et doux QU'ILS ETAIENT, ses cris devenaient menaçants). Pourquoi y trouve-t-on deux marqueurs de prédication seconde ? De plus, la relative paraît a priori redondante, dans la mesure où l'adjectif peut apparaître seul, sans qu'une modification sémantique soit manifeste (De tendres et doux, ses cris devenaient menaçants). La préposition de est déjà l'auxiliaire d'une prédication «secondaire » et la relative semble surajouter une prédication. Son analyse, qui se base sur un corpus de données authentiques, permet de mieux comprendre pourquoi la relative est jugée pertinente et non redondante. Cette construction complète la description des relatives prédicatives du type 3 et soulève la question d'une hiérarchie des prédications secondes à établir.

La contribution de Pierre Larrivée soutient que les quantifieurs flottants sont des attributs et qu'ils relèvent ainsi de la problématique plus générale de la prédication seconde. La caractérisation du statut grammatical de ces items conduit à explorer l'extension de la classe constituée, étendue par certaines études à ensemble, tous (les) deux, seuls. Larrivée entend montrer que l'un l'autre, eux-mêmes, eux autres notamment ont des comportements similaires. Si ces expressions ont dans l'emploi flottant une fonction d'attribut, la propriété du nominal auquel elles renvoient serait de marquer la situation de l'intégralité des occurrences de l'ensemble, situation qui peut être quantitative, présentative ou contrastive, distributive, globalisante ou réflexive (Les invités ont pour quelques-uns d'entre eux condamné la comtesse). On les considérera donc comme des attributs quantitatifs. Les réflexions de Larrivée montrent que la question des attributs mériterait d'être reconsidérée dans sa totalité, puisque le type 3 n'inclut traditionnellement que les attributs d'objet directs et indirects.

Les contributions reprises dans ce numéro analysent de manière détaillée certains types de constructions à prédication seconde identifiés dans des études antérieures et les comparent à d'autres structures qui ne sont pas considérées (traditionnellement) comme telles. Elles soulèvent la question de savoir s'il est vraiment possible et nécessaire de regrouper sous l'étiquette commune de prédication seconde des constructions aussi hétérogènes ; ne devrait- 
on pas plutôt réserver ce terme à un ensemble plus cohérent de constructions qui auraient toutes strictement un ancrage nominal et qui présenteraient au moins quatre des traits exposés ci-dessus, quitte à désigner l'ensemble plus large et plus flou regroupant l'ensemble des tours répertoriés au début de cette introduction simplement par l'appellation de prédications 'secondaires'?

\section{Bibliographie}

AARTS, B., 1992, Small Clauses in English, Berlin - New York, Mouton De Gruyter.

BERRENDONNER, A. \& REICHLER-BEGUELIN, M.-J., 1989, « Décalages : Les niveaux de l'analyse linguistique », Langue française, 81, p. 99-125.

BLANCHE-BENVENISTE, C., 1983, «La complémentation verbale : valence, rection et associés », Recherches sur le français parlé, 3, p. 57-98.

Blanche-BenVEnISTE, C., 1998, «L'usage prédicatif secondaire des participes passés », Prédication, assertion, information, Forsgren \& alii (éds), Uppsala, Acta Universitatis Upsaliensis, p. 43-56.

BLANCHE-BENVENISTE, C. \& alii, 1990, Le français parlé, études grammaticales, Paris, CNRS.

Blanche-Benveniste, C. \& CADDEO, S., 2000, «Préliminaires à une étude de l'apposition dans la langue parlée », Langue française, 125, p. 60-70.

BORILlo, A. (à paraître dans les actes du colloque Représentations du sens linguistique III, Bruxelles, 3-5 novembre 2005), «Quelques formes de prédication seconde appliquées à l'expression de relations temporelles ».

CADDEO, S., 2002, «Prédication et apposition », www.up.univ-mrs.fr/delic/papiers/Caddeo-2002praxiling.pdf

CADIOT, P.\& FuruKAWA, N. (éds.), 2000, « La prédication seconde », Langue française, 127.

CADIOT, P., 2000, «La préposition comme connecteur et la prédication seconde », Langue française, 127, p. $112-125$.

COMBetTES, B., 1998, Les constructions détachées en français, Paris, Ophrys.

Delorme, B. \& LefEUVRE, F., 2003, « De la prédication seconde à la prédication autonome », Verbum, XXVI, p. 281-297.

ERIKSSON, O., 1993, Phrase française. Essai d'un inventaire de ses constituants syntaxiques. Göteborg, Acta Universitatis Gothoburgensis.

ERnST, T.,2001, The Syntax of Adjuncts, Cambridge, Cambridge University Press.

FORSGREN, M., 1991, «Éléments pour une typologie de l'apposition en linguistique française », Actes du XVIIIe congrès International de Linguistique et de Philologie Romanes, Université de Trèves, Tübingen, Max Niemeyer Verlag, p. 597-611.

FoRSGREN, M., 1993, «L'adjectif et la fonction d'apposition : observations syntaxiques, sémantiques et pragmatiques », L'Information grammaticale, 58, p. 15-21.

FORSGREN, M., 2000, «Apposition, attribut, épithète : même combat prédicatif ? », Langue française, 125, p. $30-45$.

FuRUKAWA, N., 1996, Grammaire de la prédication seconde, Paris - Louvain-la-Neuve, Duculot.

GAATONE, D., 1988, «Cette coquine de construction. Remarques sur les trois structures affectives du français », Travaux de linguistique, 17, p. 159-175.

GoEs, J., 1999, L'adjectif. Entre nom et verbe, Paris - Louvain-la-Neuve, Duculot.

GREvisSe, M. \& GoOSSE, A., 1991, Le bon usage, Paris - Louvain-la-Neuve, Duculot. (édition refondue par André Goosse).

HALMØY, O., 2003, Le gérondif en français. Paris, Ophrys.

HAVU, E., 2004, « L'interprétation des constructions participiales appositives », La Linguistique, 40, p. 65-82. 
HAVU, E. \& PIERRARD M., 2004, «La prédication seconde en français : définition et extension du domaine », Linguistics Today - Facing a Greater Challenge (incl. CD Rom), Piet van Sterkenburg (éd), John Benjamins Publishing Company, Amsterdam /Philadelphia, CD-Rom: contributions IS7_EvaHavuAndMichelPierrard_LaPredicationSecondeEnFrancais.pdf

HAVU, E. \& PIERRARD M., 2006, «Le détachement est-il une propriété basique de la prédication seconde ? , L'Information grammaticale, 109, 20-26.

HAVU, E. \& PIERRARD M., 2007, «Observations sur la syntaxe des prédications secondes et des constructions attributives », Trotter, D. (éd.) Actes du XXIVe Congrès de Linguistique et de philologie romanes (Aberystwyth $1^{\text {er }}-6$ août 2004), 1, p. 199-208.

JESPERSEN, O., 1937, Analytic Syntax, New York, Holt, Rinehart and Winston.

KortMAnN, B., 1991, Free Adjuncts and Absolutes in English, London - New York, Routledge.

LAMBRECHT, K., 2002, «Topic, focus and secondary predication. The French presentational relative construction », Beyssade, C. et alii (éds), Romance Languages and Linguistic Theory, Amsterdam / Philadelphia, John Benjamin's Publishing Company.

LAGAE, V., 1998, Les constructions en 'de'+adjectif. Typologie et analyse, Leuven, Presses Universitaires de Louvain.

LEEMAN, D., 2000, « Compléments circonstanciels ou appositions ? », Langue française, 125, p. 18-29.

Melis, L. (éd.), 1988, « La prédication seconde », Travaux de linguistique, 17.

MELIS, L., 2000, « L'infinitif de narration comme prédication seconde », Langue française, 127, p. 36-48.

MulleR, C., 2000, «Les constructions à adjectif attribut de l'objet, entre prédication seconde et complémentation verbale », Langue française, 127, p. 21-35.

Neveu, F., 1998, Études sur l'apposition, Paris, Honoré Champion.

NeVEU, F., 2000, «Quelle syntaxe pour l'apposition ? Les types d'appariement des appositions frontales et la continuité référentielle », Langue française, 125, p. 106-124.

Neveu, F., 2000, «L'apposition : concepts, niveaux, domaines - Présentation », Langue française, 125, p. 317.

Neveu, F., 2002, «Du prédicat second à la clause - Sur le rang syntaxique de quelques types de détachements », Charolles, P. \& alii (éds), Actes du colloque international «Y a-t-il une syntaxe au-delà de la phrase ? », 21-22 septembre 2000, Université Paris III, Verbum 24, 1-2, p. 129-140.

NeVEU, F., 2004, «Support et référenciateur de l'adjectif dans le système appositif - Sur l'interprétation des prédicats détachés », Caen, Université de Caen/CNRS, Presses Universitaires de Caen, p. 337-356.

NoAILLY, M., 2000, «Apposition, coordination, reformulation dans les suites de deux GN juxtaposés », Langue française, 125 , p. 46-59.

PIERRARD M., 2001, «Paramètres pour l'interprétation et le classement des constructions à attribut de l'objet », Kronning, H. \& alii (éds.), Langage et référence. Mélanges offerts à Kerstin Jonasson à l'occasion de ses soixante ans, Uppsala, Acta Universitatis Upsaliensis, p. 497-507.

PIERRARD M., 2002, «Attribut de l'objet et verbe vecteur d'attribution : la place des constructions à valeur causative », Lowe, R. (éd.), Le système des parties du discours. Sémantique et syntaxe, Laval, Les Presses de l'Université Laval, p. 358-369.

RIEGEL, M., 1985, L'adjectif attribut, PUF, Paris.

RIEGEL, M., 1991, « Pour ou contre la notion grammaticale d'attribut de l'objet : critères et arguments », De Gaulmyn, M.-M. \& Rémi-Giraud, S. (éds.), A la recherche de l'attribut, Lyon, Presses Universitaires de Lyon, p. 99-118.

RIEGEL, M. \& alii , 1999 (1ère éd. 1994), Grammaire méthodique du français, Paris, PUF.

TOBBACK, E., 2005, Les constructions à attribut de l'objet et le marquage de la prédication seconde, Thèse de doctorat, Gand, Université de Gand.

WiLliams, E., 1975, «Small Clauses in English », Kimball, J. (éd), Syntax and Semantics, 4, New York, Academic Press, p. 249-273.

Williams, E., 1983, « Against Small Clauses », Linguistic Inquiry, 14 (2), p. 287-308.

WILliams, E., 1994, Thematic Structure in Syntax, Cambridge, Massachusetts, The MIT Press. 
Intro Préd2

WILMET, M., 1997, «L'apposition : une fonction à réestimer », Kleiber, G. \& Riegel, M. (éds), Les formes du sens. Études de linguistique française, médiévale et générales offertes à Robert Martin. Paris - Louvain-LaNeuve, Duculot, p. 413-422.

WILMET, M., 2007, Grammaire critique du français, Hachette -Duculot, Bruxelles, De Boek \& Larcier. 